\title{
碳碳复合材料的水射流钻孔技术研究
}

\author{
蔡志刚 陈晓川 王 迪 鲍劲松 \\ (东华大学机械工程学院 上海 201620)
}

\begin{abstract}
摘要: 针对磨料水射流加工碳碳复合材料的冲孔过程, 结合 Fluent 分析高压磨料水射流的射流压力、靶距对碳碳复合材料冲 孔深度的影响; 利用 ANSYS/ Ls-dyna 有限元分析软件建立磨料和水的射流冲击模型, 针对射流压力是冲孔分层的的主要因 素进行仿真, 得出材料失效时最大的应力和整个射流过程中应力的变化规律, 由最大应力所在位置的层间 $y$ 向位移变化确定 分层情况; 根据仿真结果进行相应的试验研究, 并将仿真结果与实验结果进行对比分析, 验证了射流仿真模型的可行性和有 效性。
\end{abstract}

关键词: 磨料水射流; 碳碳复合材料; ANSYS/Ls-dyna; 数值模拟

中图分类号: TH162

\section{Research on Water Jet Drilling Technology for Carbon-carbon Composites}

\author{
CAI Zhigang CHEN Xiaochuan WANG Di BAO Jinsong
}

(College of Mechanical Engineering, Donghua University, Shanghai 201620)

\begin{abstract}
For punching process of carbon-carbon composite processed by abrasive water jet, combined with Fluent analysis of the impact of high pressure abrasive water jet pressure and target distance on carbon-carbon composite punching depth; by Using ANSYS/Ls-dyna Finite element analysis software the impact model of abrasive and water jet is established. To simulate with that the jet pressure is the main factor of punching and stratification, obtaining the maximum stress when the material loses efficacy and the change regulation of stress through the whole jet process, The stratification by the adjacent layer in the $y$-direction displacement changes of the position of maximum stress is defined. To conduct the corresponding experimental study according to the simulation result, the simulation results and experimental results are compared to verify the feasibility and effectiveness of the jet simulation model.
\end{abstract}

Key words: abrasive water jet; carbon-carbon composite; ANSYS/Ls-dyna; numerical simulation

\section{0 前言}

碳碳复合材料是一种先进的碳纤维及其织物增 强的碳基体复合材料, 是轻质高效结构设计的理想 材料, 也属于难加工材料。目前碳纤维复合材料已 广泛应用于航空航天、汽车、造船等领域的大型结 构件中。

钻孔是碳碳复合材料后加工工艺中一个极为重 要的工序, 用常规方法加工出来的制孔精度和制孔 质量难以保证。作为迅速发展起来的磨料高压水射 流已经成为近十几年来发展最快的特种加工技术之 一, 特别适合对微小零件进行切割和精密钻孔, 有 着广阔的应用前景 ${ }^{[1]}$ 。磨料水射流将纯水用超高压 加压器, 使水的压力达到 $280 \sim 400 \mathrm{MPa}$ 左右, 用

20180301 收到初稿, 20180719 收到修改稿
高速水射流加速磨料(石榴石)粒子, 混合后从割枪 直径为 $0.2 \sim 0.4 \mathrm{~mm}$ 的细小喷嘴射出, 射流速度可 达 $800 \sim 1000 \mathrm{~m} / \mathrm{s}$, 冲击工件去除材料。

国内外对磨料水射流加工的研究成果卓著, 但 尚缺少关于磨料水射流加工复合材料机理和工艺 参数的相关研究 ${ }^{[2]}$ 。在磨料水射流加工方面主要集 中在粗加工和半精加工方面, 而且在对复合材料和 脆性材料加工的研究中, 多部分研究停滞于实验室 研究, 在工程运用上还需要很多更深入的研究 ${ }^{[3]}$ 。 近几年来, 不少学者针对复合材料的加工问题, 提 出了不同的方法: 鲍永杰等 ${ }^{[4]}$ 采出聚晶金刚石(Poly crystalline diamond, $\mathrm{PCD}$ )刀具加工; KAMLESH 等 ${ }^{[5}$ 通过试验了解了磨料水射流的切削参数对碳 纤维增强材料的钻孔过程中的分层程度的影响, 并 优化处理了参数; ZHANG 等 ${ }^{[6]}$ 通过在复合材料和 脆性材料上加工钻削深小孔的试验, 创建了经验模 型, 预测钻孔深度; JOHN 等 ${ }^{[7]}$ 使用传统钻孔和水 
射流钻孔碳纤维材料研究了其疲劳特性, 发现加工 时间长会引起的分层裂纹开始蔓延; 也有学者采 用数值模拟的方法进行分析 ${ }^{[8]}$; 李根生等 ${ }^{[9]}$ 运用流 固耦合理论, 提出并建立了高压水射流冲击过程 的数值分析模型; 张成光等 ${ }^{[10]}$ 采用 ANSYS Fluent 对磨料射流进行瞬态 CFD 仿真, 建立了射流加工 塑性材料的去除模型; 苗新刚等 ${ }^{[11]}$ 利用 Ls-dyna 有限元软件对一些常见高硬脆材料的水射流冲击 过程进行了仿真。这些主要是针对钻孔时出现的 分层情况, 出口撕裂等对刀具提出的一些改进方 法, 提高孔的加工精度和质量, 以及采用数值模拟 的方法仿真射流的过程, 而对磨料射流加工碳碳复 合材料钻孔时的工艺参数研究并不完善。采用水射 流技术可以显著降低传统机械加工的成本, 提高加 工效率。

本文通过 ANSYS/Ls-dyna 软件, 建立磨料和水 的射流冲击复合材料模型, 对射流加工过程采用流 固耦合进行仿真, 对高压水射流冲击过程中流体压 力和复合材料内部应力进行数值计算, 找到射流压 力影响复合材料分层的应力变化规律, 可以减少大 量试验带来的问题, 并将仿真结果与对应的试验结 果进行对比分析, 验证射流仿真模型的正确性和有 效性。

\section{1 射流冲击建模}

\section{1 冲击模型的建立}

复合材料具有各向异性、层间剪切等特性, 建 模的正确性对仿真有很大影响, 本文通过 ANSYS 软件建立射流加工碳碳复合材料的几何模型。在 ANSYS 中选用 Solid164 实体单元进行建模, 对复 合材料的厚度及铺层角度方向进行设置, 复合材 料板的铺层方式及角度 $\left(0 / 90^{\circ} / 45^{\circ} /-45^{\circ} / 0\right)$ 如图 1 所 示。试验中复合材料板厚度 $4.5 \mathrm{~mm}$, 由于射流水刀 直径 $0.4 \mathrm{~mm}$, 相比工件来说很小, 所以建模体积大 小为长 $20 \mathrm{~mm}$, 宽 $20 \mathrm{~mm}$ 和厚 $6 \mathrm{~mm}$, 并赋予模型

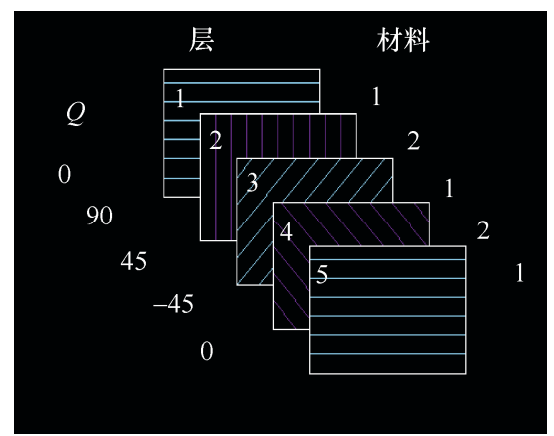

图 1 复合材料的铺层角度方向
材料本身的本构方程、密度、弹性模量等参数。模 拟冲击的复合材料为 $\mathrm{T} 300$, 其相关的力学参数如表 1 所示。

\section{表 1 复合材料 $\mathrm{T} 300$ 的力学参数}

\begin{tabular}{lc}
\hline \multicolumn{1}{c}{ 名称 } & 数值 \\
\hline 密度 $\rho /\left(\mathrm{g} / \mathrm{cm}^{3}\right)$ & 1.62 \\
泊松比 $\mu$ & 0.33 \\
弹性模量 $E / \mathrm{GPa}$ & 135 \\
纵向切变模量 $/ \mathrm{GPa}$ & 3.32 \\
纵向剪切强度 $/ \mathrm{MPa}$ & 123 \\
纵向弯曲强度 $/ \mathrm{MPa}$ & 1500 \\
纵向弯曲弹性模量 $/ \mathrm{GPa}$ & 102 \\
层间剪切强度 $/ \mathrm{MPa}$ & 84 \\
\hline
\end{tabular}

将建好的复合材料模型进行网格的划分及边 界指定, 为保证网格质量以同时减少计算时间, 对射流入口和工件中心部分局部加密处理 ${ }^{[12]}$, 对 底面进行约束, 对其他面需要建立无反射边界 条件。

复合材料模型建好后再建立磨料和水刀的模 型, 水刀射流直径为 $0.4 \mathrm{~mm}$, 将射流水刀模型完 全塑性分析, 屈服应力设为零, 假设水射流以速 度 $v$ 冲击复合材料, 则该过程建立的三维物理模 型如图 2 所示。在实际加工过程中, 水刀喷嘴结 构和尺寸基本都是固定的, 改变的是射流压力和 水刀移动的速度。压力越大, 射流的流速就越高, 去除工件的能力也越强, 所能达到的加工深度也 越深。但对于复合材料来说, 加工过程中射流压 力的变化会造成孔内部产生分层缺陷, 加工的孔 不能达到技术要求。因此, 本文通过结合 Fluent 和 Ls-dyna 仿真软件对射流冲击复合材料过程进 行仿真, 发现射流压力、靶距对射流冲孔的影响 规律, 以减少大量试验带来的成本和工作量, 找 到合适的加工工艺参数, 实现水射流技术成功加 工碳碳复合材料。

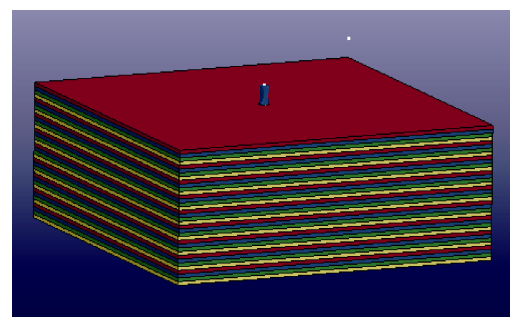

图 2 复合材料板网格划分和射流模型

\section{2 模型参数与设置}

水射流经喷嘴高速射出对工件产生冲击作用, 流体介质为水和磨料的两相流耦合, 初始时水相体 积分数为 0.9 , 磨料体积分数 0.1 , 入口为射流压力 
入口, 按公式(1)Bernoulli 方程转换为其对应的喷嘴 出口速度; 出口为射流压力出口, 为标准大气压; 其它面均被视为无滑移壁面。两相流材料特性设置: 水密度 $998 \mathrm{~kg} / \mathrm{m}^{3}$, 黏度 $8 \times 10^{-4} \mathrm{~kg} / \mathrm{m}^{3} \cdot \mathrm{s}$, 磨料颗粒 为石榴石, 硬度为 7.5 , 粒度为 $80 \#$, 密度为 $3.4 \sim$ $4.3 \mathrm{~g} / \mathrm{cm}^{3}$, 黏度 $1 \times 10^{-5} \mathrm{~kg} / \mathrm{m}^{3} \cdot \mathrm{s}$, 其他部分计算参数 可根据仿真软件默认要求进行设置。在非淹没条件 下进行固液两相流耦合冲孔过程仿真分析。

$$
v=44.7 \sqrt{P}
$$

式中, $v$ 是射流出口速度, $P$ 为射流压力。

\section{2 控制方程与边界条件}

\section{1 水射流控制方程与边界条件}

\subsection{1 水射流控制方程}

水射流的控制方程包括连续性方程、能量方程 和 $k-\varepsilon$ 方程 ${ }^{[13]}$, 这三个方程作为高压水射流射流流 场的数值模型, 在水射流数值仿真计算和射流压力 在材料内部应力分布状态的计算中常用到。其方程 组的形式如下所述。

I . 连续性方程

$$
\frac{\partial \rho_{\overline{u i}}}{\partial x_{i}}=0
$$

II. 能量方程

$$
\rho \overline{u_{j}} \frac{\partial \overline{u_{i}}}{\partial x_{j}}=-\frac{\partial \bar{p}}{\partial x_{i}}+\frac{\partial}{\partial x_{j}}\left[\mu\left(\frac{\partial \overline{u_{i}}}{\partial x_{j}}+\frac{\partial \overline{u_{j}}}{\partial x_{i}}\right)-\rho \overline{u_{i} u_{j}}\right]
$$

III. $k-\varepsilon$ 方程

$$
\begin{gathered}
\rho\left(\overline{u_{j}} \frac{\partial k}{\partial x_{j}}\right)=\frac{\partial}{\partial x_{i}}\left[\left(\mu+\frac{\mu_{i}}{\sigma_{k}}\right) \frac{\partial k}{\partial x_{i}}\right]+ \\
\mu_{i} \frac{\partial}{\partial x_{i}}\left(\frac{\partial \overline{u_{i}}}{\partial x_{j}}+\frac{\partial \overline{u_{j}}}{\partial x_{i}}\right)-\rho \varepsilon \\
\rho \overline{u_{j}} \frac{\partial_{\varepsilon}}{\partial x_{j}}=\frac{\partial}{\partial x_{i}}\left[\left(\mu+\frac{\mu_{i}}{\sigma_{\varepsilon}}\right) \frac{\partial \varepsilon}{\partial x_{i}}\right]+ \\
C_{1} \frac{\varepsilon}{k} \frac{\partial \overline{u_{i}}}{\partial x_{j}}\left(\frac{\partial \overline{u_{i}}}{\partial x_{j}}+\frac{\partial \overline{u_{j}}}{\partial x_{i}}\right)-C_{2} \rho \frac{\varepsilon^{2}}{k}
\end{gathered}
$$

方程中的 $C_{1}, C_{2}, \sigma_{k}, \sigma_{\varepsilon}$ 都为经验常数, 取 值为 $C_{1}=1.44, C_{2}=1.92, \sigma_{k}=1.0, \sigma_{\varepsilon}=1.3$ 。

\section{1 .2 边界条件}

(1) 入口边界: 入口为射流压力, 按 Bernoulli 方程转换为其对应的喷嘴出口速度, 射流水刀轴线 垂直于材料表面即流固耦合面

$$
\frac{\partial \overline{u_{i}}}{\partial x_{i}}=0 \quad \overline{u_{j}}=\overline{u_{k}}=0
$$

(2) 出口边界: 出口为射流压力出口, 为标准 大气压。

(3) 固壁条件: 水刀射流喷嘴出口和材料耦合 边界为射流场的固壁条件, 设置固面为均无滑移 移动。

\section{2 复合材料控制方程}

复合材料具有各向异性，其控制方程采用 Lagrange 法 ${ }^{[12]}$ 可以表示如下。

(1) 本构方程

$$
\begin{gathered}
\sigma_{i j}=\frac{E}{1+v}\left(\frac{v}{1-2 v} \delta_{i j} \varepsilon_{v}+\varepsilon_{i j}\right) \\
\varepsilon_{v}=\varepsilon_{11}+\varepsilon_{22}+\varepsilon_{33}
\end{gathered}
$$

式中, $E$ 为弹性模量; $v$ 为泊松比; $\delta_{\mathrm{ij}}$ 为克罗内克 $\delta$ 符号。

(2) 几何方程

$$
\varepsilon_{i j}=\frac{1}{2}\left(\frac{\partial u_{i}}{\partial x_{j}}+\frac{\partial u_{j}}{\partial x_{i}}\right)
$$

式中, $\varepsilon_{\mathrm{ij}}$ 为单元应变, $u_{i}, u_{j}$ 为单元位移。

\section{3 水射流与复合材料耦合面的控制方程}

水射流与复合材料层合板进行流固耦合时要满 足两个条件。

(1) 动力平衡条件: $\boldsymbol{n} \cdot \tau_{\mathrm{f}}=\boldsymbol{n} \cdot \sigma_{\mathrm{n}}$, 其中 $\tau_{\mathrm{f}}, \sigma_{\mathrm{n}}$ 分别表示水射流和复合材料的应力矢量, $\boldsymbol{n}$ 为流固 耦合边界的法向矢量。

（2）位移相容条件: $\boldsymbol{d}_{\mathrm{f}}=\boldsymbol{d}_{\mathrm{s}}$, 其中 $\boldsymbol{d}_{\mathrm{f}}, \boldsymbol{d}_{\mathrm{s}}$ 分别表 示流体和材料位移矢量。

通过动力平衡条件把流场压力加到复合材料 上, 实现水射流与复合材料板的流固耦合求解。

\section{3 仿真分析}

在仿真过程中射流直径、射流角度保持不变的情 况下, 改变冲孔加工过程中的水刀压力、水刀移动速 度及靶距参数, 分析其对加工孔质量的影响规律。运 用 ANSYS/fluent 有限元分析软件对高压水刀切割复 合材料钻孔的固液两相流耦合分析, 采用正交试验的 方法进行仿真, 确定合适的压力范围和最佳靶距, 以 及分析各个参数对加工结果的影响; 射流压力分别取 值为 $280 \mathrm{MPa} 、 320 \mathrm{MPa} 、 350 \mathrm{MPa} 、 375 \mathrm{MPa} 、 400 \mathrm{MPa}$ 、 $425 \mathrm{MPa} 、 450 \mathrm{MPa}$, 射流靶距为 $3 \mathrm{~mm}$, 切割速度为 $100 \mathrm{~mm} / \mathrm{min}$, 射流角度为 $90^{\circ}$ 。

把射流仿真模型导入到 Fluent 中, 加载后进行 迭代计算, 结果收玫。在水射流仿真的过程中, 对 不同的射流压力值进行仿真, 可以得到射流压力与 切割深度的关系, 如图 3 所示; 在不同的射流压力 
下, 材料的去除深度不一样, 由图 3 的数据可知在 复合材料板厚为 $6 \mathrm{~mm}$ 适用压力在 $300 \sim 350 \mathrm{MPa}$ 。 在水射流设备一定的情况下, 射流压力和射流的流 速正相关。在实际加工中, 调节最多的就是射流压 力, 适当增大射流压力, 由水流加速后的磨料射流 获得的动能也越大, 其去除加工材料的能力也越强, 加工的效果也会越好 ${ }^{[14]}$ 。当射流压力过大, 作用在 层与层间的力大于层间的剪切强度会造成分层; 当 射流压力低于某一临界值时, 磨料射流的冲蚀动能 就会不足, 无法将工件材料去除, 就会出现啃边、 撕裂、纤维脱落等缺陷。

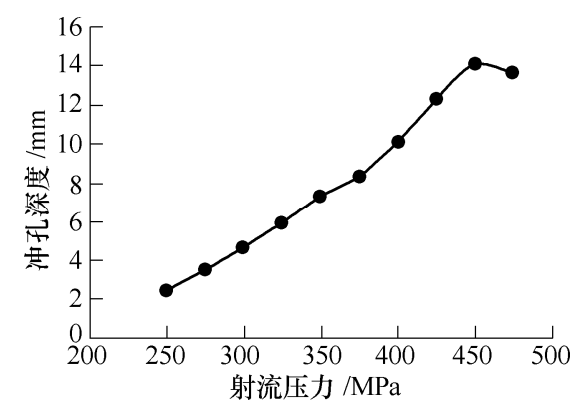

图 3 射流压力与切割深度的关系

射流靶距也是冲孔加工过程中一个重要的参 数, 是磨料射流喷嘴出口处到工件加工面之间的距 离。对不同靶距下仿真与试验其他工艺参数的设置 如下: 射流压力取值为 $320 \mathrm{MPa}$, 射流靶距为 $1 \mathrm{~mm}$ 、 $3 \mathrm{~mm} 、 5 \mathrm{~mm} 、 7 \mathrm{~mm} 、 9 \mathrm{~mm} 、 11 \mathrm{~mm} 、 13 \mathrm{~mm}$, 切 割速度为 $100 \mathrm{~mm} / \mathrm{min}$, 射流角度为 $90^{\circ}$ 。

同样, 在仿真的过程中, 只改变射流靶距这一 参数进行仿真, 可以得出靶距与工件材料去除深度 的关系, 如图 4 所示。

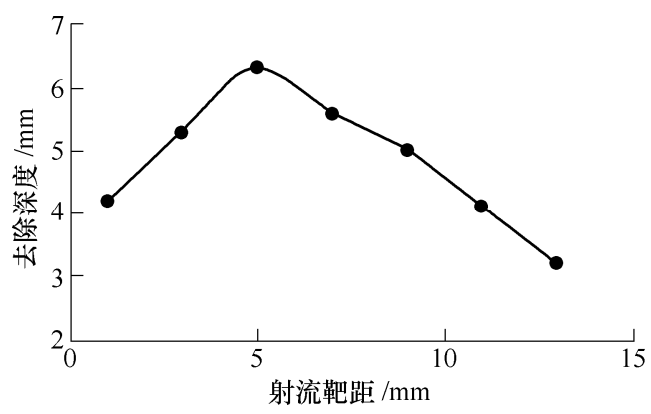

图 4 射流靶距与切割深度的关系

当射流靶距不断增大时, 工件材料的冲孔深度 先不断增加, 达到一定的射流靶距之后又开始减小。 射流靶距太大, 到达工件时射流的动能就衰减很多, 会造成射流穿透不过工件形成盲孔或有毛刺出现; 靶距太近, 射流反溅严重, 不利于冲孔。因此根据 仿真数据, 在其他加工工艺参数不变的条件下, 最 佳的射流靶距应该在 4 6 $\mathrm{mm}$ 之间。在改变射流压
力和靶距的数值仿真中, 可得出在射流压力范围在 $300 \sim 320 \mathrm{MPa}$, 喷距为 $5 \mathrm{~mm}$ 是射流冲孔复合材料 较为合适的工艺参数。

在 ANSYS/Ls-dyna 里就射流压力进行仿真分 析, 通过对复合材料模型射流区域细化网格, 定义 强度失效准则的方法, 对射流的过程进行仿真模拟, 并分析射流过程应力的大小对层间位移的变化的影 响, 得到射流过程中材料失效时最大的应力和水射 流刚接触材料时材料表面的受力情况, 以及复合材 料在整个射流过程中应力的变化, 通过查看最大应 力位置所在的层间位移的变化确定是否有分层情 况。其两相流射流冲孔压力 $320 \mathrm{MPa}$ 不同时刻冲蚀 情况仿真效果图如图 5 所示。

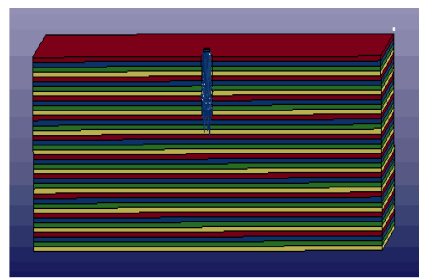

(a) 开始时刻

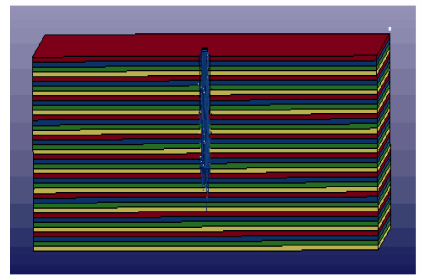

(b) 中间时刻

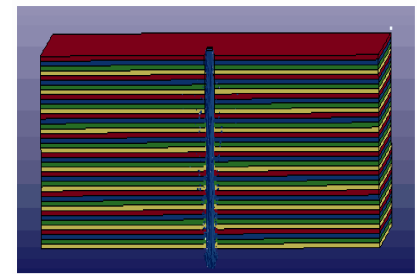

(c) 穿透时刻

图 5 射流冲孔不同时刻冲蚀情况

射流侵切的过程中, 材料受到的冲击应力是在 不断变化的, 复合材料射流冲孔过程中的最大等效 应力曲线如图 6 所示, 从图 7 可看出在网格单元

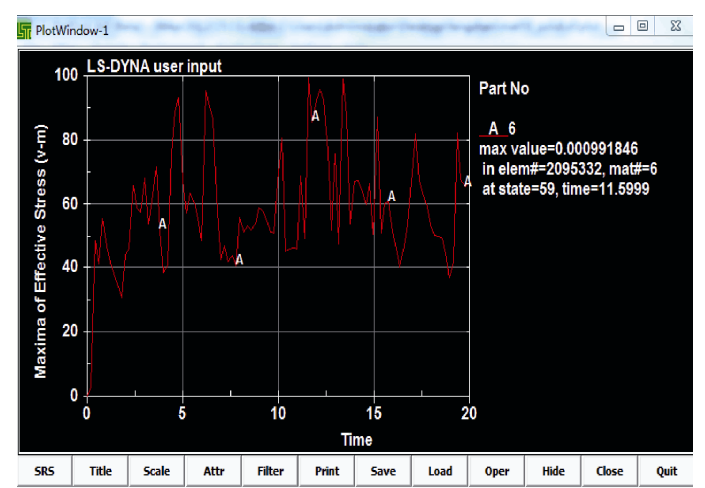

图 6 冲孔过程中最大等效应力曲线图 
2095332 有最大的应力值为 $99.2 \mathrm{MPa}$, 确定这个单 元所在层的位置, 查看相邻层间的位移的变化, 确 定是否有分层。

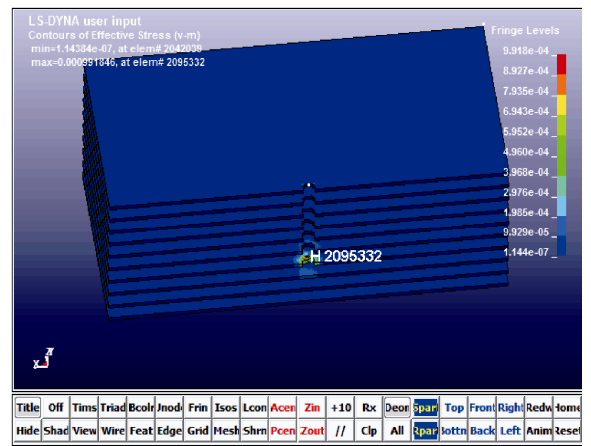

图 7 最大应力所在单元位置和应力力情况

通过查看最大应力所在层相邻层间位移的变化 情况, 如图 8、9 所示, 在射流冲击复合材料层合板 时, 由于受到的冲击力大小变化造成层间应力过大 产生分层。由图 8 可知, 在 $320 \mathrm{MPa}$ 射流压力时最 大应力处位移的大小变化极小, 相比于孔径 $6 \mathrm{~mm}$ 层合板的两层承受较大拉伸应力, 单元拉伸位移失 效范围远小于孔径大小, 可认为在射流过程中复合

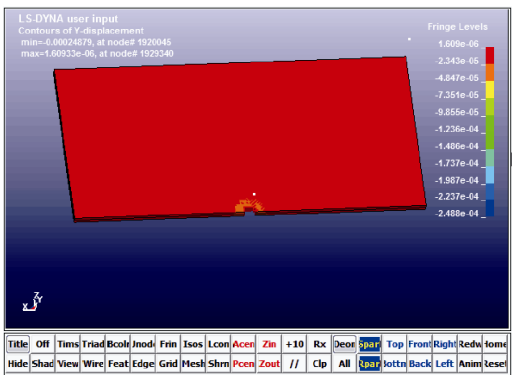

图 8 射流压力 $320 \mathrm{MPa}$ 最大应力所在相邻层 $y$ 向位移

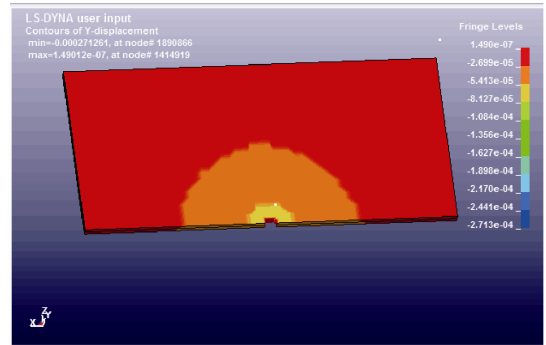

(a) $280 \mathrm{MPa}$

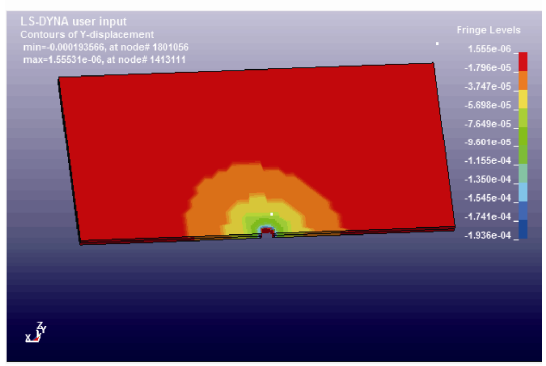

(b) $350 \mathrm{MPa}$

图 9 射流压力 $280 \mathrm{MPa}$ 和 $350 \mathrm{MPa}$ 最大应力 所在层 $y$ 向位移
材料分层位移相比大位移的变化可不考虑相关的影 响。如图 9a、 $9 \mathrm{~b}$ 分别为 $280 \mathrm{MPa}$ 和 350MPa 压力下 的最大应力对应的位移，由于射流压力的作用，产 生的应力过大使层间位移的变化较大，呈椭圆状向 外扩散并减小，更易造成分层。

在水射流冲击作用下复合材料内部产生很大的 应力, 并且应力的分布具有一定的规律性, 在水射 流与材料的冲击接触区域产生的应力最大，然后呈 椭圆状向外扩散，并逐渐减小。因此材料失效总是 从射流冲击接触区域开始的, 并逐步向外扩散, 这 与实际加工过程是一致的。

\section{4 试验与结果分析}

为了验证射流仿真过程中的射流压力、靶距与 孔质量是否分层之间的联系，针对上述仿真得到的 加工工艺参数进行磨料水射流冲孔试验, 然后通过 超声扫描显微镜(SAM300)对实际加工孔进行分层 缺陷的检测。

考虑到水射流设备对射流压力的实际可调节范 围，在射流加工中，采用正交试验的方法做了多组 试验验证射流压力对孔质量的影响 ${ }^{[15]}$, 选择射流压 力在 $260 \sim 400 \mathrm{MPa}$ 之间进行试验, 冲孔射流喷距 5 $\mathrm{mm}$, 横移速度 $100 \mathrm{~mm} / \mathrm{s}$, 垂直射流冲孔, 孔径大 小在 $6 \mathrm{~mm}$, 并记录多组试验数据。对射流加工的孔 进行 SAM 分层缺陷检测成像结果如图 10 所示, 可 以看到: 白色高亮区域即为分层缺陷的 SAM300 基 本成像特征, 遇到空隙界面(分层)时会发生的强超 声反射回波。

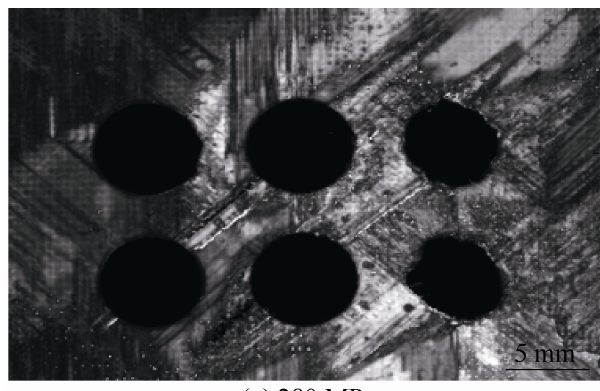

(a) $280 \mathrm{MPa}$

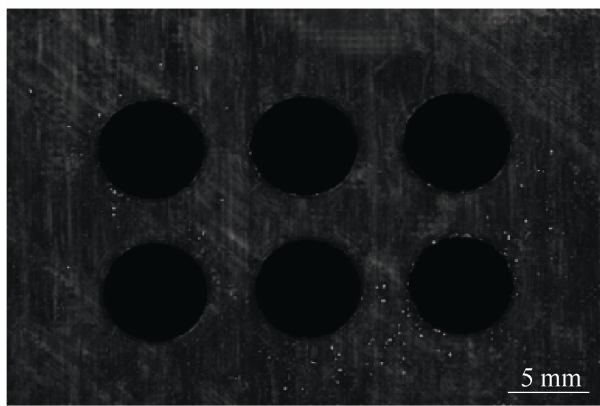

(b) $320 \mathrm{MPa}$ 


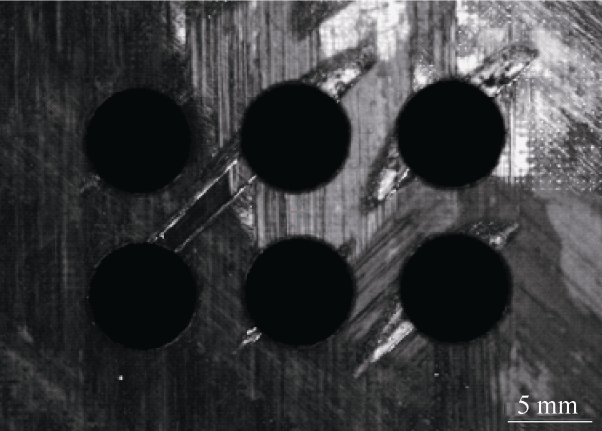

(c) $350 \mathrm{MPa}$

图 10 射流冲孔分层缺陷的 SAM 检测结果

从 SAM 检测结果可以看出, 在射流压力为 320 $\mathrm{MPa}$ 检测图 $10 \mathrm{~b}$ 上, 白色高亮区域基本没有, 成像 特征一致, 说明没有分层缺陷发生; 而在射流压力 $280 \mathrm{MPa}$ 检测图 $10 \mathrm{a}$ 上有明显的白色高亮区域, 说 明内部有分层缺陷, 甚至在压力较低时右列孔型不 圆; 在 $350 \mathrm{MPa}$ 检测图 10c 上也有明显的白色高亮 区域, 内部也有分层缺陷。

在磨料射流冲孔复合材料时, 射流压力对材料 是否造成分层和孔径质量好坏是一个很重要的影响 因素。通过仿真确定一定范围的加工射流压力和靶 距, 再根据仿真数据进行试验加工出来的孔与仿真 分析误差小, 吻合度很高, 具有很高的可信度。

\section{5 结论}

（1）通过 Fluent 流体分析软件, 采用正交试验 的方法仿真了射流压力、靶距与冲孔深度之间的关 系, 以及分析了在加工工艺中射流压力是主要的影 响因素, 建立了工艺参数与加工深度的关系: 随射 流压力的增大, 切割深度也越大, 但射流压力过大, 作用在层与层间的力大于层间的剪切强度易造成 分层。

(2) 针对射流压力是冲孔分层的的主要因素进 行仿真, 根据材料失效时最大的应力和整个射流过 程中应力的变化规律, 通过查看最大应力所在位置 的层间 $y$ 向位移变化确定分层情况, 这样可以减少 大量试验带来的问题 。

(3) 利用 ANSYS/Ls-dyna 有限元分析软件对复 合材料的射流冲孔加工过程进行了仿真, 并将试验 结果与仿真结果进行了对比分析, 发现仿真与试验 吻合度很高, 验证了仿真模型的可行性与有效性, 体现了其在工程上的应用价值。

\section{参 考 文 献}

[1] 李增强, 赵佩杰, 宋宇轩, 等. 微磨料水射流加工技术
研究现状 [J]．纳米技术与精密工程，2016，14(2): 134-144.

LI Zengqiang, ZHAO Peijie, SONG Yuxuan, et al. Research status of micro abrasive water jet machining technology[J]. Nanotechnology and Precision Engineering, 2016, 14(2): 134-144.

[2] 王伟. 高压磨料水射流切割碳纤维复合材料的试验研 究[D]. 哈尔滨: 哈尔滨理工大学, 2015.

WANG Wei. Study on the experiment of carbon fiber composite materials with high - pressure AWJ cutting[D] Harbin: Harbin University of Science and Technology, 2015.

[3] 曹国强, 张睿. 高压水射流研究现状及应用[J]. 沈阳航 空航天大学学报, 2017, 34(3): 1-16.

CAO Guoqiang, ZHANG Rui. Review on high pressure water jet[J]. Journal of Shenyang Aerospace University, 2017, 34(3): 1-16.

[4] 鲍永杰, 高航. 碳纤维复合材料构件加工缺陷与高效加 工对策[J]. 材料工程, 2009(S2): 254-259.

BAO Yongjie, GAO Hang. Drilling disfigurements formation analysis and solution of CFRP[J]. Journal of Materials Engineering, 2009(S2): 254-259.

[5] KAMLESH P, RAMESH S, SANDIP P, et al. Delamination characterization and comparative assessment of delamination control techniques in abrasive water jet drilling of CFRP[J].Procedia Manufacturing , 2016, 5: 521-535.

[6] ZHANG Fenglian , JING Zhu. An experimental study of drilling small and deep blind holes with an abrasive wtiter jet[J]. Modern Manufacturing Engineering, 2009, 5: 87-90.

[7] JOHN M, HABIBA B, FAWAZ Z, et al. Influence of drilling and abrasive water jet induced damage on the performance of carbon fabric epoxy plates with holes[J]. Composite Structures, 2017, 163: 257-266.

[8] KARNIK S R, GAITONDE V N, CAMPOS R J. Delamination analysis in high speed drilling of carbon fiber reinforced plastics (CFRP) using artificial neural network model [J]. Materials \& Design, 2008, 29 (9): 1768-1776.

[9] 李根生, 廖华林, 黄中伟, 等. 超高压水射流作用下岩 石损伤破碎机理[J]. 机械工程学报，2009，45(10): 285-293.

LI Gensheng, LIAO Hualin, HUANG Zhongwei, et al. Rock damage mechanisms under ultra-high pressure water jet impact[J]. Journal of Mechanical Engineering, 2009, 45(10): 285-293..

[10] 张成光, 张勇, 张飞虎, 等. 磨料水射流加工去除模型 研究[J]. 机械工程学报, 2015, 51(7): 188-196. 
ZHANG Chengguang, ZHANG Yong, ZHANG Feihu, et al. Study on Removal Model of Abrasive Waterjet Machining. Journal of Mechanical Engineering, 2015, 51(7): 188-196.

[11] 苗新刚, 武美萍, 缪小进. 磨料水射流冲蚀工件仿真与 试验研究[J]. 液压气动与密封, 2016(1): 44-47.

MIAO Xingang, WU Meiping, MIAO Xiaojin. Simulation and experimental research of abrasive water jet erosion workpiece[J]. Hydraulics Pneumatics \& Seals, 2016(1): 44-47.

[12] 叶建友, 吕彦明. 水射流冲击压力最佳喷距数值仿真与 试验研究 $[J]$. 电加工与模具, 2014(5): 34-36.

YE Jianyou, LÜ Yanming. Numerical simulation and experimental research on the best jet distances of water jet impact force[J]. Electromachining \& Mould, 2014(5): 34-36.

[13] 廖华林, 李根生, 罗学创, 等. 超高压水射流冲击应力 分布规律的数值分析 [J]. 石油钻采工艺, 2004, 26(5): $8-12$.

LIAO Hualin, LI Gensheng, LUO Xuezhao, et al.
Numerical analysis of the distribution law of impact stress of ultra high pressure water jet[J]. Oil Drilling\& Production Technology, 2004, 26(5): 8-12.

[14] 叶建友, 吕彦明. 基于 FLUENT 的水射流冲击力影响 因素仿真分析 [J]. 电加工与模具, 2014(6): 50-52.

YE Jianyou, LÜ Yanming. Simulation and analysis on the influence factors of water jet impact force based on FLUENT[J]. Electromachining \& Mould, 2014(6): $50-52$.

[15] 李志凯. 碳纤维增强复合材料切削试验与仿真研究[D]. 南昌: 南昌航空大学, 2014 .

LI Zhikai. Study of cutting experiment and simulation of carbon fiber reinforced composites[D]. Nanchang : Nanchang Hangkong University, 2014.

作者简介: 蔡志刚, 男, 1991 年出生。主要研究方向为智能制造与测 控技术。

E-mail: caizhigang8023@163.com 陈晓川(通信作者), 男, 教授, 硕士研究生导师。主要研究方向为复合 材料加工、纺织机械设计、面向成本的设计、人工智能等。

E-mail: xcchen@dhu.edu.cn 